\title{
Louis-Philippe Dalembert. Entre vagabondage et humanisme, dir. D.-H. PAGEAUX
}

\section{Carminella Biondi}

\section{(2) OpenEdition}

\section{Journals}

\section{Édition électronique}

URL : https://journals.openedition.org/studifrancesi/32393

DOI : $10.4000 /$ studifrancesi.32393

ISSN : 2427-5856

Éditeur

Rosenberg \& Sellier

Édition imprimée

Date de publication : 1 août 2020

Pagination : 452-453

ISSN : 0039-2944

\section{Référence électronique}

Carminella Biondi, «Louis-Philippe Dalembert. Entre vagabondage et humanisme, dir. D.-H. PAgEaux », Studi Francesi [En ligne], 191 (LXIV | II) | 2020, mis en ligne le 01 septembre 2020, consulté le 18 septembre 2021. URL : http://journals.openedition.org/studifrancesi/32393 ; DOI : https://doi.org/10.4000/ studifrancesi.32393

Ce document a été généré automatiquement le 18 septembre 2021.

\section{(c) (i) (9)}

Studi Francesi è distribuita con Licenza Creative Commons Attribuzione - Non commerciale - Non opere derivate 4.0 Internazionale. 


\title{
Louis-Philippe Dalembert. Entre vagabondage et humanisme, dir. D.-H. PAGEAUX
}

\author{
Carminella Biondi
}

\section{RÉFÉRENCE}

Louis-Philippe Dalembert. Entre vagabondage et humanisme, dir. D.-H. PAGEAUX, Paris, L'Harmattan, 2018, «Classiques pour demain», $261 \mathrm{pp}$.

1 «En fait tu recherches un autre pays. Celui que dans tes pérégrinations en terre étrangère tu as nommé le pays-temps. Celui qu'on n'habite jamais qu'une seule fois. Comme le fleuve d'Héraclite. Cette terre d'au-delà des races et des nationalités. Deux notions vides de sens, deux hasards de l'aventure humaine. Donc absurdes». Cette citation, tirée du premier roman de Dalembert, Le Crayon du bon Dieu n'a pas de gomme (1996), est la parfaite synthèse d'une vie et d'une œuvre: une vie vagabonde qui a trouvé ses racines mouvantes, justement, dans ce lieu que l'auteur appelle un paystemps, qui suit l'écrivain dans ses déplacements, et une œuvre qui a fleuri dans le pays de sa naissance, Haïti, pour s'élargir à la mesure du monde, et s'implanter, tour à tour, surtout là où la rencontre entre les hommes est porteuse de conflits, mais aussi d'un renouveau de vie.

2 Les mélanges qu'on présente ici contribuent, dans leurs différentes approches, à éclairer ce parcours existentiel et artistique de l'écrivain et nous guident jusqu'au seuil $\mathrm{du}$ dernier roman, qui n'est pas abordé dans ce recueil car il a paru après sa publication, Mur méditerranée (2019), un roman centré sur l'actualité la plus brûlante, qui nous contraint à entrer dans la tragédie de la migration africaine vers l'Europe, dont l'Italie, et en particulier Lampedusa, où l'écrivain lui-même a vécu, représente la première étape. Ce parcours éclaire aussi le deuxième terme du sous-titre de ces mélanges, «humanisme», car c'est vers ce besoin d'embrasser l'humanité souffrante 
(«les éclopés du destin») que pointe le vagabondage de Dalembert, ce qui ne signifie pas, ainsi qu'il émerge clairement des contributions à ce recueil, que l'auteur nous propose une œuvre à thèse, mais certainement une œuvre à idées.

3 Ces mélanges sont coordonnés de main de maître par Daniel-Henri PAGEAUX, qui connaît Louis-Philippe Dalembert depuis la fin des années quatre-vingt $d u \mathrm{xx}^{\mathrm{e}}$ siècle, quand il dirigeait son mémoire de maîtrise, suivi d'un mémoire de DEA et d'une thèse de doctorat (1996). C'est grâce à cette longue fréquentation de l'écrivain et de son œuvre, qu'il a pu en retracer, dans son essai conclusif, «Persistance de l'humain»: pour une relecture de Louis-Philippe Dalembert (pp. 227-252), une synthèse éclairante, qui clôt magistralement le volume. Une conclusion qui aurait pu être aussi une parfaite introduction à la mosaïque d'essais qui le composent, auxquels l'ouverture sur un tableau d'ensemble aurait, peut-être, profité. Cependant l'organisation de la matière critique est savamment agencée pour permettre au lecteur de suivre un parcours aussi bien thématique que chronologique, et donc de lire en même temps la matière de l'écriture et son évolution. Le recueil est divisé en cinq parties, d'ampleur différente, qui réunissent les essais selon leur focalisation sur un thème ou sur une œuvre: «Vagabondages» (pp. 17-68), «L'autre face de la mer» (pp. 71-108), «Rue du Faubourg SaintDenis» (pp.111-160), "Avant que les ombres s'effacent» (pp.163-168), "Variations linguistiques» (pp. 171-253). L'ensemble est composé de treize essais, écrits pour ces mélanges ou déjà parus ailleurs, qui n'arrivent pas toujours à éviter quelques redites, mais qui représentent, tous, une contribution originale et un guide utile pour approcher une œuvre qui n'est qu'apparemment facile. Nous ne pouvons ici les analyser tous, mais ils méritent d'être au moins cités, car leur titre est déjà une petite synthèse de l'ensemble, dans lequel une place non marginale est réservée à l'écriture: Alba PESSINI, L'arpentage du monde dans l'œeuvre de Louis-Philippe Dalembert; Alessia VIGNOLI, Louis-Philippe Dalembert, "vagabond jusqu'au bout de la fatigue»; Emanuela CACCHIOLI, Départs volontaires, faux départs, retours impossibles dans l'œuvre de Louis-Philippe Dalembert; Gaëlle COREMAN, Migration clandestine vers le "premier monde» (l'Amérique, l'Europe). Les «boat people» haïtiens dans "L'Autre face de la mer" de Louis-Philippe Dalembert; Odile GANNIER, Retour au "pays-temps» de Grannie (Louis-Philippe Dalembert: "Le Crayon du bon Dieu n'a pas de gomme", "L'Autre face de la mer", "Les Dieux voyagent la nuit"); Jean-Marie THÉODAT, Autobiographie du faubourg; Steve PUIG, Ancrages et déplacements chez LouisPhilippe Dalembert: le cas de "Rue du Faubourg Saint-Denis"; Étienne ACHILLE, "Rue du Faubourg Saint-Denis" de Louis-Philippe Dalembert: de Romain Gary à Haïti ou une nouvelle cartographie pour l'idéal républicain français; Annie FOREST - Abou MANSOUR, Entre fiction et réel [à propos d'Avant que les ombre s'effacent, dernier roman de l'auteur lors de la parution des mélanges]; Hugues SAINT-FORT, L'écriture créole de Louis-Philippe Dalembert; Marcelo MARINHO, L'arbre à palabres, paysages imaginaires: regards sur une traduction brésilienne de Louis-Philippe Dalembert; Yves CHEMLA, La poésie de Louis-Philippe Dalembert; et l'essai conclusif de Pageaux dont j'ai déjà parlé.

Les titres nous indiquent que toute l'œuvre de Dalembert est ici prise en compte, avec peut-être une focalisation plus poussée sur Rue du Faubourg Saint-Denis, dont l'histoire se passe dans un quartier parisien, et qui représente donc un moment-pivot (Puig, p. 138) dans la carrière de l'écrivain, qui laisse en sous-texte Haïti pour accentuer son choix de faire dialoguer des cultures. Une caractéristique de l'œuvre de Dalembert, qui ne ressort pas des titres, mais qui a sollicité l'attention de beaucoup de contributeurs, est l'usage des épigraphes, tirées souvent de la Bible mais aussi de ses écrits antérieurs, 
ce qui tisse, avec le retour de certains personnages, un lien fécond d'un ouvrage à l'autre dans un parcours qui cartographie les espaces-temps de la narration et du vécu.

On ne saurait mieux conclure cette présentation, forcément limité et limitante, que par un passage de la préface de Daniel-Henri Pageaux sur le sens profond de l'œuvre que nous sommes invités à lire, ainsi qu'il émerge de la fouille accomplie par les essais réunis dans ces mélanges, un sens qui nous interpelle en tant que voyageurs dans un monde où, face aux frontières de plus en plus perméables, s'élèvent des murs de plus en plus solides: «L'écriture de Dalembert est un bel exemple de marche contre les ténèbres. En ce sens, son écriture est aussi, comme il a été dit, un pari sur l'homme et sur l'humain [...]. C'est cette marche sans entraves qu'on peut aussi appeler vagabondage, qui a permis au gavroche caribéen [...] de maîtriser symboliquement, métaphoriquement le monde dans lequel il lui a été donné de vivre: une tâche, un exemple, une leçon qui font de Louis-Philippe Dalembert un classique non seulement pour demain, mais aussi pour aujourd'hui» (pp. 12-13). 